\title{
A Novel Linear Precoding Scheme for Multi-user Generalized Space Modulation System
}

\author{
Shunran WANG ${ }^{1, a}$, Yanhui LU ${ }^{2, \text { b }}$, Shouyi YANG, Panpan MIAO \\ ${ }^{1}$ School of Information Engineering, Zhengzhou University, Zhengzhou 450001, China \\ ${ }^{2}$ School of Information Engineering, Zhengzhou University, Zhengzhou 450001, China \\ aemail: 1422356093@qq.com, bemail: ieyhlu@zzu.edu.cn
}

Keywords: multi-user; generalized spatial modulation; dimension reduction; Partial Linear Precoding

\begin{abstract}
In the Multi-user Generalized Spatial Modulation (MU-GSM) System, as channel gain matrix isn't in full rank, conventional linear precoding algorithms can't be directly applied in this system. Given this, a method called Multi-user Generalized Spatial Modulation Partial Linear Precoding (MU-GSM-PLP) is proposed for MU-GSM system. MU-GSM system is established in this method that the antennas at the transmitter are grouped and Generalized Spatial Modulation (GSM) is adopted in each antenna group. Then, dimensional reduction is made on channel gain matrix as well as transmitting signal matrix, and the equivalent channel gain matrix and transmitting signal matrix are obtained. Finally, Partial Linear Precoding (PLP) precoding is carried out by utilizing the equivalent channel gain matrix. The simulation results show that this method have a better error performance and higher system capacity.
\end{abstract}

\section{Introduction}

MIMO is the key technology in wireless communication nowadays. It improves system capacity and reliability without adding bandwidth [1-2]. However, in MU-GSM system, interference is introduced and requirement for synchronization is higher, moreover, it has higher hardware complexity. Spatial Modulation (SM) effectively solves the above problems, SM actives one antenna at transmitter and maps partial information to conventional digital modulation constellation point and the rest to space dimensions generated by antenna indexes [3-4]. In this way, interference can be completely avoided. Generalized Spatial Modulation (GSM) activates at least two antennas at transmitter, which relieves the limitation that the number of activated antenna in Spatial Modulation system must be 2 of power. Meanwhile, it increases diversity gain and excellently improves spectral efficiency, but the most, it inevitably introduces channel interference [5-6].

Among existing technologies, J. Wu researched multi-user MIMO system with SM precoding. After block diagonalization, this system is equal to multiple parallel single-user MIMO systems, and then inter-channel interference is eliminated through SM [7]. But in the MU-GSM system, as there are at least two activated antennas so that interference can't be avoided. Owing to channel gain matrix is not a full rank matrix, the generalized inverse does not exist, (Partial Linear Precoding) PLP cannot be directly used in MU-GSM system for removing interference.

A Multi-user Generalized Spatial Modulation Partial Linear Precoding (MU-GSM-PLP) method is provided in this paper. MU-GSM system is decomposed into multiple independent single-user GSM systems by grouping the antennas of transmitter and then dimensional reduction is produced according to the feature that there exist silence antennas in GSM on channel gain matrix and transmitting signal matrix. It can be seen that the transmitting signal information and channel state information of activated antennas is abstracted by dimensionality reduction matrix. Finally, through PLP method, destructive part of interference is retained. In this paper, after transmitting signal matrix processed by precoding matrix, due to destructive interference among users and channels is eliminated as well as the constructive interference is kept, the detection of antenna indexes and transmitting bits will be more accurate by employing Maximun Liklihood (ML) or Maximum Ratio Combining (MRC) method at receiver, as a result, error performance and system capacity is further 
improved.

\section{Design of MU-GSM system}

A downlink MU-GSM system is showed in Fig.1, where $N_{t}$ stands for the number of the Base Station (BS) antennas, $K$ is the number of users. We assume that user $i \in\{1, \cdots, K\}$ has $M_{i}$ antennas and $N_{r}=\sum_{i=1}^{K} M_{i} \leq N_{t}$ [8]. As shown in Fig.1, transmitting antennas are uniformly divided and each group adopts GSM with $N_{t} / K$ antennas to serves a specific use, the $i^{\text {th }}$ group serving user $i$ is assumed. We assume that the $i^{\text {th }}$ user has the same number of antennas as the active antennas in the $i^{\text {th }}$ group antenna and the active antenna number of the $i^{\text {th }}$ group antenna simultaneously transmit the same data symbol.

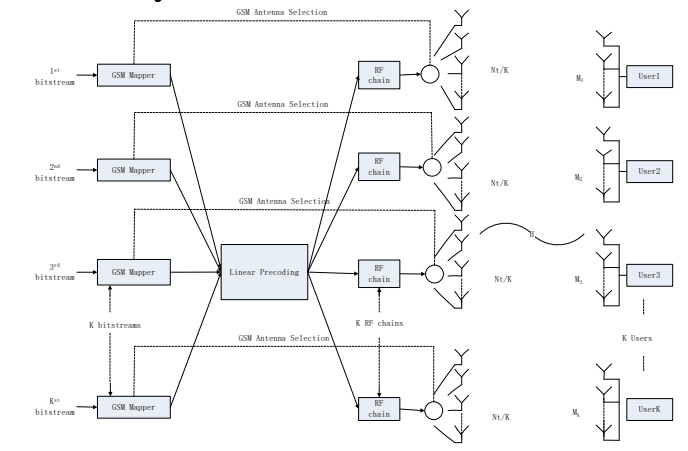

Fig. 1 - Generalized spatial modulation system module

Contrary to SM, in a GSM system, more than one transmitting antennas are activated and active antennas transmit the same symbol. Hence, a chain of antenna combinations are formed as spatial constellation points [9]. We assume that $N_{u}$ represents the number of active antennas of each antenna group. The received signal is showed below:

$$
\boldsymbol{y}=\left[\begin{array}{c}
\boldsymbol{y}^{(1)} \\
\boldsymbol{y}^{(2)} \\
\vdots \\
\boldsymbol{y}^{(K)}
\end{array}\right]=f \cdot \boldsymbol{H P}\left[\begin{array}{c}
\boldsymbol{x}^{(1)} \\
\boldsymbol{x}^{(2)} \\
\vdots \\
\boldsymbol{x}^{(K)}
\end{array}\right]+\left[\begin{array}{c}
\boldsymbol{n}^{(1)} \\
\boldsymbol{n}^{(2)} \\
\vdots \\
\boldsymbol{n}^{(K)}
\end{array}\right]
$$

Where $f$ is the power scale factor. $\boldsymbol{y}^{(i)} \in \mathbb{C}^{M_{i} \times 1}$ and $\boldsymbol{n}^{(i)} \sim C N\left(0, \sigma_{n}^{2}\right)$ represent the $i^{\text {th }}$ user's received signal and additive white Gaussian noise (AWGN), respectively. The symbol from the $i^{t^{\text {th }}}$ antenna group is $\boldsymbol{x}^{(i)}=\left[\begin{array}{llllllllllll}0 & \cdots & s_{q}^{\left(l_{1}, i\right)} & 0 & \cdots & s_{q}^{\left(l_{2}, i\right)} & 0 & \cdots & s_{q}^{\left(l_{N_{u}(i)}, i\right)} & 0 & \cdots & 0\end{array}\right]^{\boldsymbol{H}} \in \mathbb{C}^{N_{t} / K \times 1}$, where $N_{u}{ }^{(i)}$ indicates the active antenna number in the $i^{\text {th }}$ antenna group. $s_{q}^{\left(l_{j}, i\right)}\left(i=1, \cdots, K ; q=1, \cdots M_{\text {mod }} ; j=1, \cdots, N_{u}{ }^{(i)}\right)$ is the $q^{\text {th }}$ system in the traditional $M_{\text {mod }}$ order signal modulation constellation, emitted from the $j$ antenna in the $i^{\text {th }}$ group. $\boldsymbol{H}$ is the Rayleigh flat fading channel and transmitter perfectly knows the whole channel station information (CSI). $\boldsymbol{H}$ can be expressed in the form of block matrices:

$$
\boldsymbol{H}=\left[\begin{array}{cccc}
\boldsymbol{H}_{1}^{(1)} & \boldsymbol{H}_{2}^{(1)} & \cdots & \boldsymbol{H}_{K}^{(1)} \\
\boldsymbol{H}_{1}^{(2)} & \boldsymbol{H}_{2}^{(2)} & \cdots & \boldsymbol{H}_{K}^{(2)} \\
\vdots & \vdots & \ddots & \vdots \\
\boldsymbol{H}_{1}^{(k)} & \boldsymbol{H}_{2}^{(k)} & \cdots & \boldsymbol{H}_{K}^{(k)}
\end{array}\right]
$$


Where, $\boldsymbol{H}_{n}^{(i)} \in \mathbb{C}^{M_{i} \times\left(N_{t} / K\right)}$ represents the channel information between the $n^{\text {th }}$ antenna group to the $i^{\text {th }}$ user. $\boldsymbol{P} \in \mathbb{C}^{N_{t} \times N_{r}}$ is the precoding matrix. $\overline{\boldsymbol{X}}=\boldsymbol{P \boldsymbol { X }}$ is written as the transmitted symbol, which satisfies $\mathrm{E}\left[\|\overline{\boldsymbol{x}}\|^{2}\right] \leq P_{T}$, where $P_{T}$ indicates BS's transmit power, $\mathrm{E}[\bullet]$ and $\|\cdot\|$ denote the expectation and the Euclidean norm, respectively. We assume that each independently data symbol has unit variance, therefore the total transmit power constrain can be recorded as: $\operatorname{Tr}\left(\boldsymbol{P P}^{\mathrm{H}}\right) \leq \mathrm{P}_{\mathrm{T}}$.

\section{MU-GSM-PLP Design}

In a MU-GSM system, the columns and rows in channel gain matrix and transmitted signal matrix which are corresponding to the location of silence antennas are zero vectors, respectively. Then, we will create dimensionality reduction matrix according to the above nature. We assume that the number of receiving antenna of the $i^{\text {th }}$ user is the same as the number of active antenna number of $i^{\text {th }}$ group antenna. Without precoding method, the received signal can be written as:

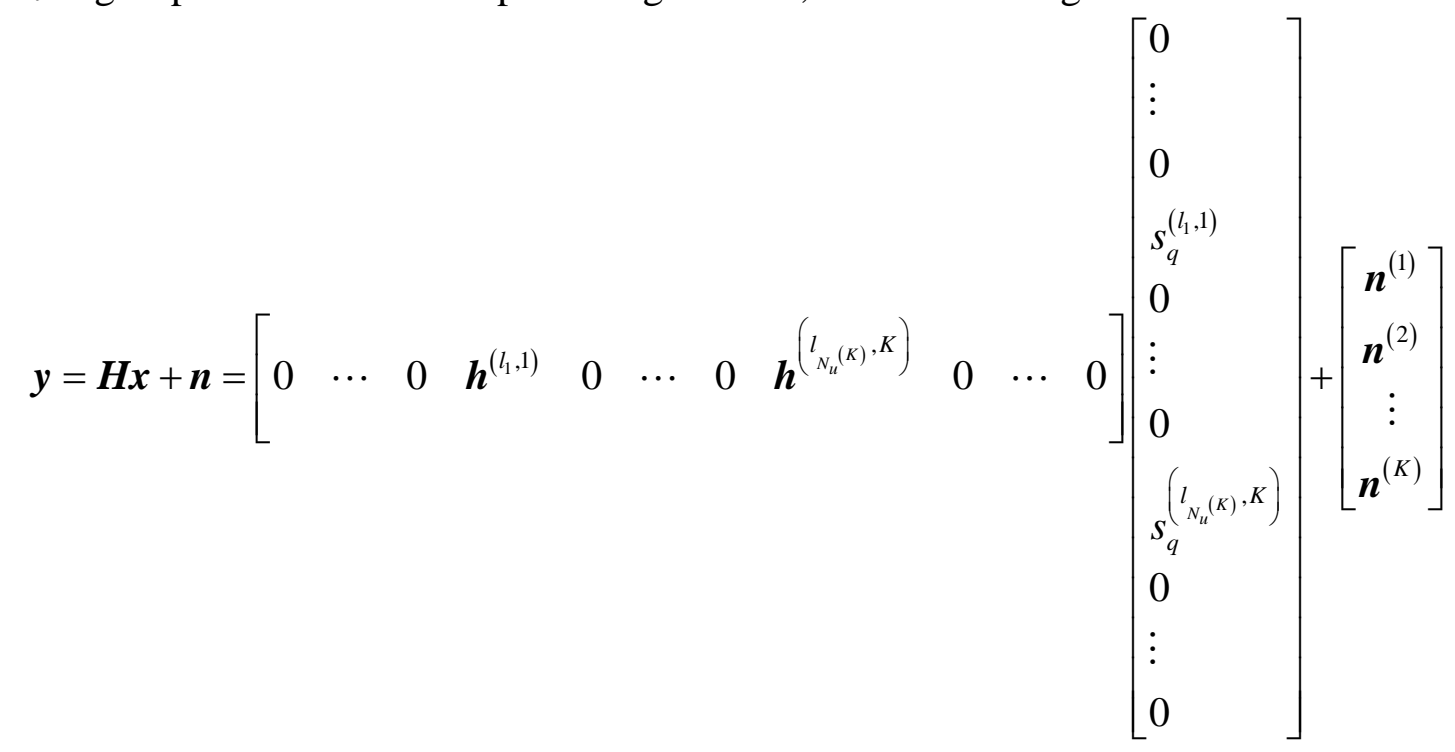

Where $\boldsymbol{h}^{\left(l_{1}, 1\right)}$ is the channel information between the $j^{\text {th }}$ antenna and the $i^{\text {th }}$ antenna group, Since only active antennas transmit information, can the received signal be further written as:

$$
\left[\begin{array}{c}
y_{1} \\
\vdots \\
y_{N_{r}}
\end{array}\right]=\left[\begin{array}{lll}
\boldsymbol{h}^{\left(l_{1}, 1\right)} & \cdots & \boldsymbol{h}^{\left({ }^{N_{u}(K)}, K\right)}
\end{array}\right]\left[\begin{array}{c}
s_{q}^{\left(l_{1}, 1\right)} \\
\vdots \\
\left({ }^{\left(l_{N_{u}}(K), K\right)}\right)
\end{array}\right]+\left[\begin{array}{c}
\boldsymbol{n}^{(1)} \\
\boldsymbol{n}^{(2)} \\
\vdots \\
\boldsymbol{n}^{(K)}
\end{array}\right]
$$

The equivalent channel matrix and the equivalent information matrix is showed below:

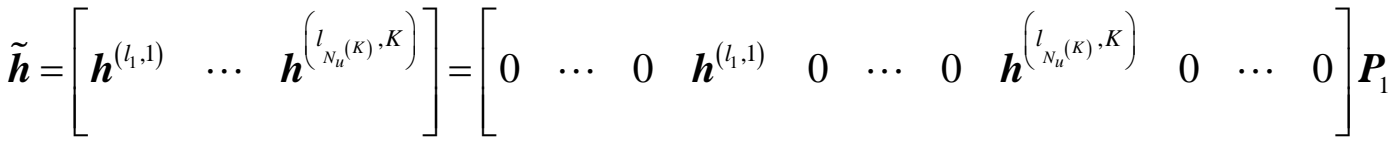

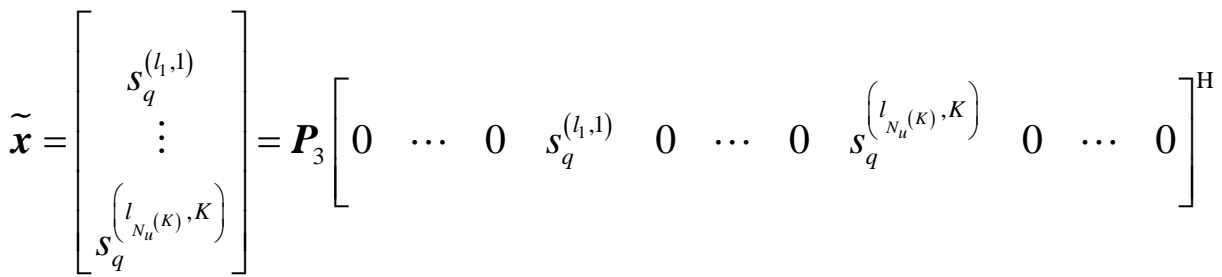

Therefore, the composition of $\boldsymbol{P}_{1}$ is that at the $l\left(l_{j}, i\right)$-th row, the $\left(\sum_{i=0}^{1} N_{u}{ }^{(i)}+j\right)-$ th column is 
1 , and the rest elements are 0 , recorded as $\boldsymbol{P}_{1}\left(l\left(l_{j}, i\right), \sum_{k=1}^{i-1} N_{u}{ }^{(k)}+j\right)=1$, where $l\left(l_{j}, i\right)$ acts the index of the $j^{\text {th }}$ antenna in the $i^{\text {th }}$ group. Similarly, $\boldsymbol{P}_{3}\left(\sum_{k=1}^{i-1} N_{u}{ }^{(k)}+j, l\left(l_{j}, i\right)\right)=1$. MU-GSM-PLP matrix can be written as $\boldsymbol{P}=\boldsymbol{P}_{1} \boldsymbol{P}_{2} \boldsymbol{P}_{3}$, where $\boldsymbol{P}_{2} \in \mathbb{C}^{N_{r} \times N_{r}}$ is PLP precoding matrix. $\boldsymbol{P}_{2}$ can be written as:

$$
\boldsymbol{P}_{2}=\widetilde{\boldsymbol{H}}^{\mathrm{H}}\left(\widetilde{\boldsymbol{H}} \widetilde{\boldsymbol{H}}^{\mathrm{H}}\right)^{-1} \boldsymbol{R}_{c}
$$

The precoding scheme of PLP can be described below:

$$
\left\{\begin{array}{l}
D_{\mathrm{R}}=\operatorname{Re}\left(s_{q}{ }^{(i)} \cdot \rho_{u, n}{ }^{(i)}\right) \cdot \operatorname{Re}\left(s_{q}{ }^{(i)}\right) \\
D_{\mathrm{I}}=\operatorname{Im}\left(s_{q}{ }^{(i)} \cdot \rho_{u, n}{ }^{(i)}\right) \cdot \operatorname{Im}\left(s_{q}{ }^{(i)}\right)
\end{array}\right.
$$

Where, $D_{\mathrm{R}}$ and $D_{\mathrm{I}}$ represent real part and imaginary part of product that desired symbol is multiplied by the interference, respectively. $S_{q}{ }^{(i)} \cdot \rho_{u, n}{ }^{(i)}$ represents the interference. The $S_{q}{ }^{(i)}$ is the target symbol, $\rho_{u, n}{ }^{(i)}$ is the element at the $u$-th row the $n$-th column of the cross-correlation matrix $\boldsymbol{R}^{(i)}=\widehat{\boldsymbol{H}}^{(i)} \widehat{\boldsymbol{H}}^{(i) \mathrm{H}}$, where $\widehat{\boldsymbol{H}}^{(i)}$ indicates channel information between the $i^{\text {th }}$ user and the $i^{\text {th }}$ antenna group. Since the energy of the signal transmitted from the silent antennas is 0 , thus we assume that the symbol from the silent antenna is 0. The interference judgment criterions for QPSK and BPSK modulation are:

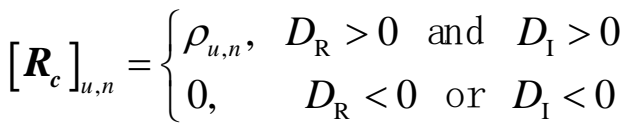

$$
\begin{aligned}
& {\left[\boldsymbol{R}_{c}\right]_{u, n}= \begin{cases}\rho_{u, n}, & D_{\mathrm{R}}>0 \\
0, & D_{\mathrm{R}}<0\end{cases} }
\end{aligned}
$$

Through the above analysis, precoding matrix and transmitted power normalization factor are exhibited below:

$$
\begin{aligned}
\boldsymbol{P}_{P L P} & =\boldsymbol{P}_{1} \boldsymbol{P}_{2} \boldsymbol{P}_{3}=\boldsymbol{P}_{1} \widetilde{\boldsymbol{H}}^{\mathrm{H}}\left(\widetilde{\boldsymbol{H}} \widetilde{\boldsymbol{H}}^{\mathrm{H}}\right)^{-1} \boldsymbol{R}_{\boldsymbol{c}} \boldsymbol{P}_{3} \\
f_{P L P} & =\sqrt{P_{T} / \operatorname{Tr}\left(\boldsymbol{P}_{P L P} \boldsymbol{P}_{P L P}^{\mathrm{H}}\right)}
\end{aligned}
$$

The received symbol of the $u$-th user's can be acquired and the average SINR of the $u$-th users can be obtained::

$$
\begin{aligned}
\begin{aligned}
& y_{P L P, u}=f_{P L P} \cdot \boldsymbol{H} \boldsymbol{P}_{1} \boldsymbol{P}_{2} \boldsymbol{P}_{3} \boldsymbol{x}+n_{u} \\
&=f_{P L P} \cdot \boldsymbol{R}_{c, u} \bar{x}_{u}+n_{u} \\
&=f_{P L P} \cdot\left(\bar{x}_{u} \cdot \rho_{u, u}+\sum_{n \neq u} \bar{x}_{n} \cdot \rho_{u, n}\right)+n_{u} \\
& \operatorname{SINR}_{P L P, u}=\left\{\mathrm{E}\left[\left\|f_{P L P} \cdot \bar{x}_{u} \cdot \rho_{u, u}+E\right\|^{2}\right]+E\left[\left\|f_{P L P} \cdot \sum_{n \neq u} \bar{x}_{n} \cdot \rho_{u, n}\right\|^{2}\right]\right\} / \sigma_{\mathrm{n}}^{2}
\end{aligned}
\end{aligned}
$$

\section{Test results}

This section simulates the performance of MU-GSM-PLP by employing Monte Carlo method. We assume that the channel is flat fading channel, the noise is zero-mean additive white Gaussian noise, the number of users is $K=6$, the number of transmit antennas and activated antennas among transmit antennas in each group is $N_{t} / M=7$ and $N_{u}^{(i)}=2$, respectively. 


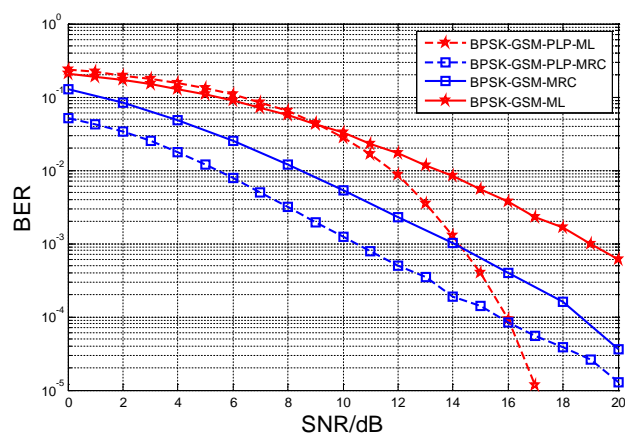

Fig. 2 -BER performance comparison of MU-GSM with MU-GSM-PLP using ML detector and MRC detector

Figure 2 simulates the performance of Bit Error Ratio (BER) of GSM with BPSK modulation varied with SNR. It can be observed that along with the increase of SNR, BER reduced gradually. The dash line and square nodes curve indicate error performance curve of MU-GSM system detected by ML and detected by MRC, respectively. The better error performance will be gotten when utilizing MU-GSM-PLP, as MU-GSM-PLP retains the constructive interference. The MU-GSM system, which employs MU-GSM-PLP method and ML will get the same error performance with that adopts MRC detection and that employs MU-GSM-PLP method and MRC detection at SNR equals $14 \mathrm{~dB}$ and $16 \mathrm{~dB}$, respectively. It suggests that ML detection will achieve more accurate detection results with smaller noise energy.

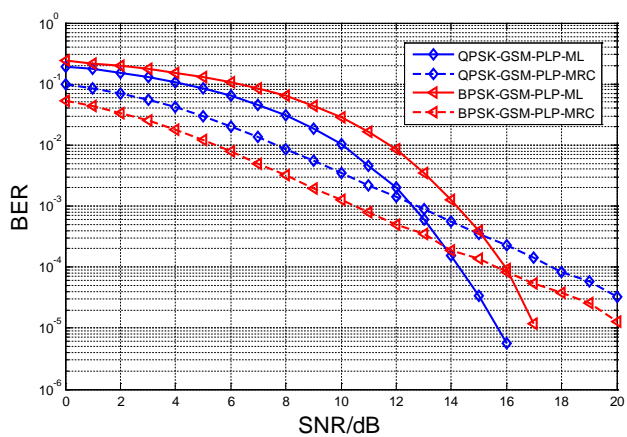

Fig. 3 -BER performance comparison of MU-GSM with MU-GSM-PLP using BPSK-GSM modulation and QPSK-GSM detector

Figure 3 simulates the performance of Bit Error Ratio (BER) varied with SNR used BPSK modulation and QPSK modulation respectively. We can observe that when MRC detection is used the change of error performance is gentler and better error performance will be achieved by adopting BPSK modulation. This is because the detection of antenna indexes has nothing to do with the change of noise energy on the condition that MRC detection are adopted and each signal contains 2bit in QPSK modulation, while, there is only 1bit for each signal of BPSK modulation, respectively. When using ML detection, error performance of system with QPSK modulation is better. As ML detection is largely affected by noise energy, the slop variation of error performance curve is bigger.

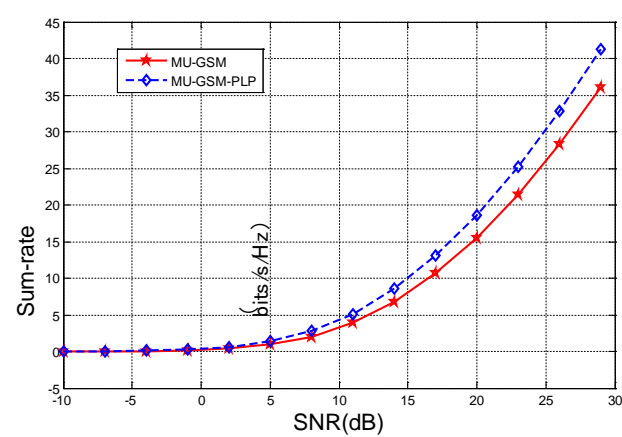

Fig. 4 -sum-rate performance comparison of MU-GSM with MU-GSM-PLP and MU-GSM without MU-GSM-PLP 
The sum-rate at receiver can be expressed as $R=K \cdot \log _{2}(1+\operatorname{SINR})$. Figure 4 simulates the sum-rate of GSM system with MU-GSM-PLP method and that without MU-GSM-PLP. Simulation conditions are the same as shown in figure 2. The curve with diamond node represents sum-rate performance curve of GSM system with MU-GSM-PLP method. It can be seen that performance of sum-rate of MU-GSM-PLP has significantly improved owing to the use of linear precoding which can be able to take advantage of constructive interference.

\section{Conclusion}

In this paper, we have proposed MU-GSM-PLP method for MU-GSM system. Here, MU-GSM system is decomposed into many independent single-user GSM systems; then the dimensions of channel gain matrix and transmitting information matrix are reduced according to characteristics of GSM system. PLP precoding method contain retains the constructive interference and eliminate the destructive interference of MU-GSM system. MU-GSM-PLP linear precoding can effectively enhance system capacity and error performance.

\section{Acknowledgement}

This work was supported in part by the Program for New Century Excellent Talents in University under Grant No.NCET-12-0699, National Natural Science Foundation of China under Grant No.61271421, Program for Young Teachers in Colleges and University in Henan Province under Grand No.2011GGJS-002, and Henan Province Major science and technology project under Grant No.112102210507.

\section{References}

[1] Marco Di Renzo, Harald Haas ; Peter M. Grant, Spatial modu- lation for multiple-antenna wireless systems: a survey [M], IEEE, Communications Magazine,2011, 49(12) 182 -191.

[2] Jan Mietzner, Robert Schober, Lutz Lampe, Wolfgang H. Gerstacker, Peter A. Hoeher, Multiple-antenna techniques for wireless communications- A comprehensive literature survey[J], IEEE Communications Surveys \& Tutorials, 2009,11(2) 87- 105.

[3] R. MESLEH, H. HAAS, S. SINANOVI'C,C. W. AHN, S. YUN, Spatial modulation[J]. IEEE Transaction on Veh- icular Technology, 2008, 57(4) 2228 -2241.

[4] Raed Mesleh, Harald Haas ; Peter M. Grant, Trellis Coded Spatial Modulation [J].IEEE Transaction on Wireless Communication, 2010, 9(7) 2349-2361.

[5] LIU Wenlong, WANG Nan, JIN Minglu, Spare detection algorithm for generalized spatial modulation system [J]. Signal Processing, 2013,29(9) 1233-1237,(in chinese)

[6] S. P. Alaka, T. Lakshmi Narasimhan, A. Chockalingam, Generalized spatial modulation in indoor wireless visible light communication[C]// 2015 IEEE Global Communications Conference, SAN Diego, CA, 2015.

[7] Wu Jiali, The precoding technology for multi-user MIMO spatial modulation, College of Physical Science and Technology Central China Normal University, 2014.

[8] Kaige Yang and Christos Masouros, On The optimal number of antennas for power efficient generalized spatial modulation[C]// Computer Aided Modelling and Design of Communication Links and Networks (CAMAD), Guildford, United Kingdom, 2015.

[9] Xueru Li, Yan Zhan, Limin Xiao, Xibin Xu, Jing Wang1, A novel precoding scheme for downlink multi- user spatial modulation system[C]// 2013 IEEE 24th International Symposium on Personal, Indoor and Mobile Radio Communications, London, United Kingdom, 2013. 